\title{
Polirradiculoneuropatia periférica desmielinizante associada ao uso de carbamazepina em cão: relato de caso
}

\author{
[Demyelinating peripheral polyradiculoneuropathy associated with the use of carbamazepine \\ in dog: case report] \\ S.M. Caramalac ${ }^{1}$, S.M. Caramalac ${ }^{1}$, T.D. Fernandes ${ }^{2}$, M.I.P. Palumbo ${ }^{2 *}$ \\ ${ }^{1}$ Aluno de pós-graduação - Universidade Federal do Mato Grosso do Sul - Campo Grande, MS \\ ${ }^{2}$ Universidade Federal do Mato Grosso do Sul - Campo Grande, MS
}

\begin{abstract}
RESUMO
Diversos fármacos são utilizados no tratamento da epilepsia e, assim como outros medicamentos, podem induzir a ocorrência de efeitos adversos, alguns tão graves que geram a necessidade de descontinuidade e substituição da terapia. A carbamazepina pode levar a alterações nos sistemas cardiovascular, respiratório e neurológico, sendo descritos na literatura casos de indução de miastenia gravis como distúrbio neuromuscular. Este estudo relata o caso de um cão que desenvolveu polirradiculoneuropatia desmielinizante, tendo como provável desencadeante a terapia com carbamazepina. O paciente apresentou tetraplegia, ausência de reflexos espinhais nos quatro membros, fraqueza cervical, diminuição do reflexo palpebral bilateral e esforço respiratório. A eletroneuromiografia demonstrou sinais de desmielinização. Este, portanto, é o primeiro relato de associação entre carbamazepina e polirradiculoneuropatia desmielinizante em cão.
\end{abstract}

Palavras-chave: eletroneuromiografia, epilepsia, fármacos, polirradiculoneuropatia

\begin{abstract}
Different drugs are used in the treatment of epilepsy and, like other drugs, may induce the occurrence of adverse effects, some of them so severe that the drug must be discontinued and replaced. Carbamazepine may lead to changes in the cardiovascular, respiratory, and neurological systems, and cases of induction of myasthenia gravis as a neuromuscular disorder have been described in the literature. This paper reports the case of a dog that developed demyelinating polyradiculoneuropathy, probably triggered by carbamazepine. The patient presented tetraplegia, absence of spinal reflexes in the four limbs, cervical weakness, decreased bilateral eyelid reflex and respiratory effort. Electroneuromyography showed signs of demyelination. This, therefore, is the first report of association between carbamazepine and demyelinating polyradiculoneuropathy in dogs.
\end{abstract}

Keywords: electroneuromyography, epilepsy, drugs, polyradiculoneuropathy

\section{INTRODUÇÃO}

A epilepsia é desordem neurológica frequente em cães e humanos, sendo caracterizada por episódios de crises convulsivas recorrentes causadas por descargas paroxísticas de neurônios cerebrais (Volk., 2015). Em cães, o fenobarbital é o fármaco de primeira escolha para o tratamento, sendo eficaz como terapia única em até $85 \%$ dos animais (Boothe et al., 2012). Nos casos em que as crises convulsivas não são controladas, ou quando os animais se tornam refratários devido à indução da enzima microssomal hepática (p450), há necessidade de adição de outras drogas à terapia, sendo comumente realizada a associação com brometo de potássio como segunda opção (Boothe et al., 2012). Entretanto, em torno de $30 \%$ dos casos

Recebido em 14 de novembro de 2017

Aceito em 9 de março de 2018

*Autor para correspondência (corresponding author)

E-mail: mariana.palumbo@ufms.br 
não há resposta clínica eficaz, de modo que se deve buscar fármacos alternativos, como carbamazepina, gabapentina, levetiracetam, zonisamida e felbamato, mas poucos são os estudos sobre o uso dessas medicações na veterinária (Chandler, 2006).

A carbamazepina é um composto derivado do iminodibenzil, estruturalmente semelhante aos antidepressivos tricíclicos e extensivamente usada em humanos para tratamento de epilepsia, dor neuropática e transtornos afetivos (Ambrósio et al., 2002). Ela atua reduzindo a permeabilidade dos canais de sódio e cálcio voltagem-dependentes, dos neurônios envolvidos na atividade epileptiforme (Sitges et al., 2016). Contudo, esse fármaco pode induzir efeitos colaterais e tóxicos ao sistema nervoso, que se manifestam como sedação, ataxia, vertigem, diplopia, nistagmo, cefaleia, estupor e coma (Ambrósio et al., 2002). Após a interrupção da medicação, o tempo médio de internação em unidades de terapias intensivas é entre um a quatro dias, ocorrendo melhora clínica 20 a 48 horas após a ingestão (Lifshitz et al., 2000).

Polirradiculoneuropatia desmielinizante inflamatória aguda, também denominada na medicina humana de síndrome de Guillain-Barré, consiste em uma polineuropatia imunomediada, caracterizada por desmielinização das raízes nervosas ventrais e dorsais, com degeneração axonal secundária, resultando em tetraparesia/plegia flácida aguda ascendente (Stanciu et al., 2014). Os primeiros relatos desta condição em animais ocorreram em cães da raça Coonhound, após contato com a saliva de guaxinim. Entretanto, atualmente, sabe-se que outros fatores também podem ser responsáveis pelo desenvolvimento desta polineuropatia, como doenças infecciosas e reação pós-vacinal (Cumming et al., 1982).

Há relatos de associação entre transtornos neuromusculares e uso de carbamazepina, estando todos eles, entretanto, relacionados à indução de miastenia gravis (Kurian e King, 2003). O presente estudo tem como objetivo relatar o primeiro caso de polirradiculoneuropatia desmielinizante aguda associada ao uso de carbamazepina em um cão.

\section{CASUÍSTICA}

Um canino, fêmea, de um ano e oito meses de idade, castrado, pesando $5,0 \mathrm{~kg}$, da raça Lhasa Apso, foi encaminhado para atendimento clínico no Hospital Veterinário da Universidade Federal de Mato Grosso do Sul (UFMS), com histórico de tetraplegia flácida aguda havia sete dias, desenvolvida horas após administração oral de carbamazepina. Segundo relato do proprietário, o animal apresentava histórico de convulsões havia um ano, as quais não diminuíram de intensidade ou frequência com o uso de fenobarbital. O tutor foi, então, orientado a iniciar a terapia com carbamazepina (Tegretol®, suspensão oral a $2 \%$, Novartis), na dose de $5 \mathrm{mg} / \mathrm{kg}$, a cada 12 horas. Entretanto, aproximadamente duas horas após a administração da primeira dose da medicação, o animal começou a apresentar paresia de membros posteriores, que evoluiu para paralisia generalizada e fraqueza cervical ao longo de seis horas. O exame neurológico, realizado no dia do atendimento (uma semana após início do quadro clínico), evidenciou ausência dos reflexos espinhais nos quatro membros, fraqueza cervical, diminuição do reflexo palpebral bilateral, paralisia de cauda e dispneia. O nível de consciência estava normal, e não foram observadas alterações em outros nervos cranianos. As sensibilidades superficial e profunda estavam preservadas, e todos os parâmetros avaliados no exame físico geral estavam sem alteração.

Hemograma, análises bioquímicas (ALT, FA, GGT, CK, creatinina, ureia, proteína total, albumina, globulina, glicemia, colesterol, triglicérides, cálcio ionizado, fósforo, sódio e potássio), hemogasometria e análise liquórica estavam dentro dos valores de normalidade, com exceção do cálcio ionizado $(0,89 \mathrm{mmol} / \mathrm{L}$, referência: 1,15 a $1,39 \mathrm{mmol} / \mathrm{L})$. Após infusão intravenosa de $1 \mathrm{~mL} / \mathrm{kg}$ de gluconato de cálcio e normalização do exame, o animal não apresentou melhora neuromuscular. Exames sorológicos de leishmaniose DPP® (Dual Path Platform, BioManguinhos/Fiocruz) e ELISA também foram realizados no início dos sinais clínicos, sendo ambos os resultados negativos. 
Em razão da suspeita de crise miastênica, foi realizada aplicação intravenosa de $0,02 \mathrm{mg} / \mathrm{kg}$ de metilsulfato de neostigmina, 15 minutos após injeção subcutânea de $0,022 \mathrm{mg} / \mathrm{kg}$ de atropina, não havendo sinais de melhora clínica após a medicação. Uma amostra de soro foi submetida à dosagem de anticorpos antirreceptores de acetilcolina (ACh) por ELISA, e, devido à gravidade do quadro clínico, optou-se por iniciar tratamento empírico com brometo de piridostigmina (Mestinon $\AA, 1 \mathrm{mg} / \mathrm{kg}, \mathrm{VO}$, a cada 12 horas) e prednisona (Meticorten ${ }^{\circledR}, 1 \mathrm{mg} / \mathrm{kg}$, VO, a cada 24 horas, por sete dias) até a obtenção do resultado deste exame.

O paciente foi reavaliado sete dias após o início do tratamento com piridostigmina e corticosteroide, e não apresentava mais dispneia, sendo observada discreta diminuição da fraqueza de musculatura cervical (sustentava a cabeça por alguns segundos). Nesse dia, foi realizado exame eletroneuromiográfico.

Utilizou-se equipamento modelo Neuromep Micro da Neurosoft®, com banda passante de 20 a $3.000 \mathrm{~Hz}$, para estudos de condução motora, e de 20 a $10.000 \mathrm{~Hz}$, para miografias. Foram utilizados eletrodos de superfície adesivos para G1, G2 e terra e eletrodo de agulha concêntrica para miografias. $\mathrm{O}$ animal foi previamente raspado nos locais de posicionamento dos eletrodos de superfície e terra. Estudos de condução motora evidenciaram prolongamentos de latências motoras distais, reduções de amplitudes, dispersões temporais, morfologias sugerindo desmielinização e acentuadas reduções de velocidades (Fig. 1). Em membro anterior esquerdo (nervo ulnar), observou-se bloqueio de condução. As eletromiografias demonstraram atividades insercionais aumentadas, atividades espontâneas frequentes dos tipos ondas agudas positivas e fibrilações, potenciais de unidades motoras com sinais de reinervação recente em músculos paravertebrais lombares e recrutamento acentuadamente reduzido ou ausente (Fig. 2). As alterações foram compatíveis com polirradiculoneuropatia periférica desmielinizante, com sinais de degeneração axonal secundária acentuada.

\begin{tabular}{|c|c|c|c|c||c|}
\hline $\begin{array}{c}\text { Pto. } \\
\text { estim. }\end{array}$ & $\begin{array}{c}\text { Lat. } \\
\mathrm{ms}\end{array}$ & $\begin{array}{c}\text { Ampl. } \\
\mathrm{mV}\end{array}$ & $\begin{array}{c}\text { Dist. } \\
\mathrm{mm}\end{array}$ & $\begin{array}{c}\mathrm{Vel} . \\
\mathrm{m} / \mathrm{s}\end{array}$ \\
\hline \multicolumn{5}{|c|}{ Esquerda, Interósseo, Ulnar } & \\
\hline Distal & 2,9 & 0,205 & & & \\
\hline Proximal & 14,9 & 0,052 & 130 & 10,8 \\
\hline
\end{tabular}

Figura 1. Amplitudes reduzidas, acentuada redução de velocidade de condução motora. Presença de bloqueio de condução e morfologia de potenciais sugerindo desmielinização.

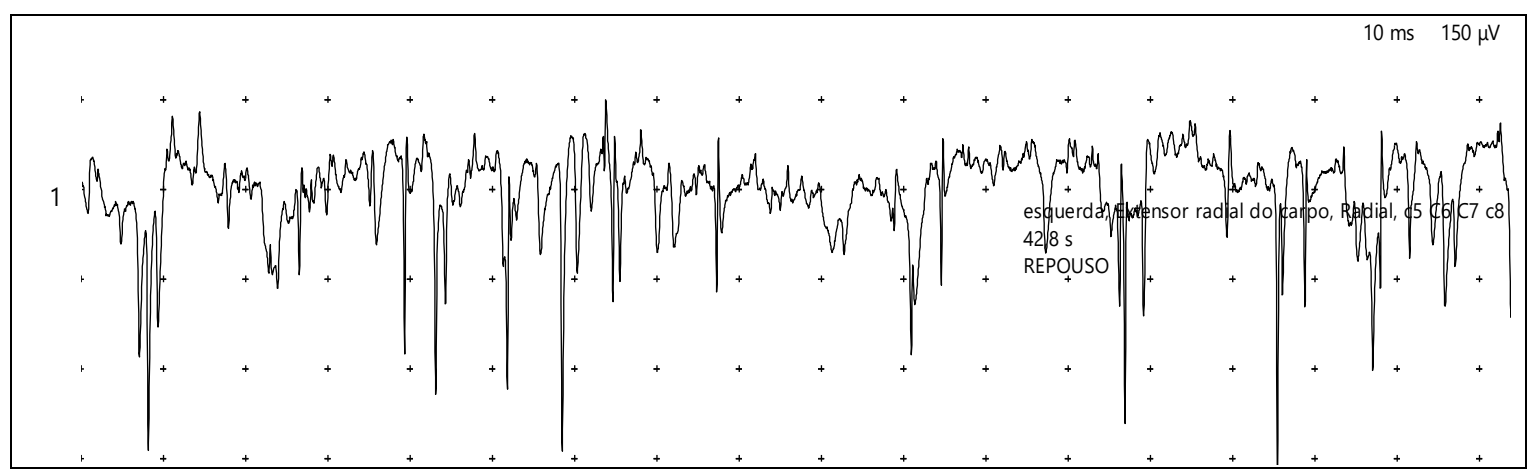

Figura 2. Traçado eletromiográfico de repouso evidenciando atividades espontâneas muito frequentes dos tipos onda aguda positiva e fibrilação. 
O proprietário foi orientado a interromper a administração da piridostigmina e a realizar redução gradual da prednisona até total suspensão, além de submeter o animal à fisioterapia intensiva. $\mathrm{O}$ paciente voltou a deambular em aproximadamente 30 dias, e o exame neurológico normalizou-se após dois meses.

\section{DISCUSSÃO}

Diversas desordens podem levar ao comprometimento do sistema nervoso periférico, apresentando, como característica comum, a redução ou a ausência de reflexos espinhais. Em cães, a tetraparesia flácida aguda está relacionada a quatro principais diagnósticos diferenciais: paralisia do carrapato, botulismo, miastenia gravis e polineuropatia periférica aguda (Añor, 2014). No presente relato, a ingestão de toxina botulínica foi descartada por não haver a possibilidade de o animal ter ingerido lixo ou carne em putrefação, além de que não houve melhora clínica dentro do tempo esperado para os casos de botulismo (Añor, 2014). Ademais, não havia histórico de presença de carrapatos, sendo descartado, portanto, o diagnóstico diferencial de paralisia do carrapato.

O paciente apresentou o quadro clínico seis horas após o início do tratamento com carbamazepina. A princípio, suspeitou-se de intoxicação, apesar de a medicação ter sido administrada na dose preconizada para a espécie. Segundo Ambrósio et al. (2002), pode ocorrer toxicidade aguda por carbamazepina em doses terapêuticas, o que acarreta sedação, ataxia, tontura, vômito, constipação e diarreia. Como o animal deste relato havia recebido uma única dose de carbamazepina e não havia apresentado nenhuma melhora clínica em sete dias após a suspensão da medicação, a possibilidade de ser um caso de intoxicação foi descartada. Além disso, não havia manifestado sinais gastrointestinais ou de sistema nervoso central.

$\mathrm{Na}$ síndrome de Guillain-Barré (humanos), o líquido cefalorraquidiano é caracterizado pela dissociação proteíno-citológica, que consiste no aumento de proteínas sem pleocitose devido à alteração na permeabilidade da barreira hematoencefálica (Sampaio et al., 2011). Cummings et al. (1982) verificaram a presença dessa alteração no líquor de três cães com polirradiculoneuropatia, semelhante ao que ocorre em humanos. O líquido cefalorraquidiano do paciente deste relato apresentava-se normal, como já foi descrito por Cuddon (2002) em pacientes nos primeiros dias da doença.

As alterações eletromiográficas dos animais com polirradiculoneuropatia são diminuição da amplitude do potencial de ação muscular composto, latência mínima da onda $\mathrm{F}$ aumentada, aumento da razão da onda $\mathrm{F}$ e diminuição da amplitude da onda F (Cuddon, 1998). O registro de potenciais de fibrilação e de ondas agudas positivas é usual e ocorre em todos os casos (Añor, 2014), sendo observado no presente relato. Segundo Feitosa et al. (2000), valores médios normais de duração da velocidade de condução nervosa distal do nervo ulnar são de $60,50 \pm 7,86 \mathrm{~m} / \mathrm{s}$. No presente relato, entretanto, a velocidade de condução motora foi em torno de $10 \mathrm{~m} / \mathrm{s}$ nos nervos estudados, o que indica grave comprometimento da condução neuronal.

Kurian e King (2003) relataram casos de pacientes humanos que desenvolveram miastenia gravis durante tratamento com carbamazepina. No presente estudo, no entanto, a concentração de anticorpos antirreceptores de Ach estava dentro do intervalo de normalidade.

Os sinais clínicos do paciente deste relato são compatíveis com polirradiculoneuropatia desmielinizante aguda, que se manifesta como paralisia muscular aguda. Segundo a literatura consultada, não foram encontrados, até então, relatos da sua associação com o uso de medicamentos.

\section{CONCLUSÃO}

Pode-se concluir que este é o primeiro relato da associação do uso de carbamazepina e do desenvolvimento de polirradiculoneuropatia periférica desmielinizante em cão. A convulsão corresponde ao distúrbio neurológico mais rotineiramente atendido na clínica de pequenos animais, sendo necessária associação de fármacos nos casos refratários ao tratamento convencional. Entretanto, é necessário cautela na utilização de terapias ainda pouco estudadas, uma vez que não se conhecem todos os seus potenciais efeitos adversos. Também se pode concluir que a avaliação eletroneuromiográfica foi essencial para o diagnóstico da polirradiculoneuropatia periférica desmielinizante. 


\section{REFERÊNCIAS}

AMBRÓSIO, A.F.; SOARES-DA-SILVA, P.; CARVALHO, C.M.; CARVALHO, A.P. Mechanisms of action of carbamazepine and its derivatives, oxcarbazepine, BIA 2-093, and BIA 2-024. Neurochem. Res., v.27, p.121-130, 2002.

AÑOR, S. Acute lower motor neuron tetraparesis. Vet. Clin. N. Am. Small Anim. Pract., v.44, p.1201-1222, 2014.

BOOTHE, D.M.; DEWEY, C.; CARPENTER, D.M. Comparison of phenobarbital with bromide as a first-choice antiepileptic drug for treatment of epilepsy in dogs. J. Am. Vet. Med. Assoc., v.240, p.1073-1083, 2012.

CHANDLER, K. Canine epilepsy: what can we learn from human seizure disorders? Vet. J., v.172, p.207-2017, 2006.

CUDDON, P.A. Acquired canine peripheral neuropathies. Vet. Clin. N. Am. Small Anim. Pract., v.32, p.207-249, 2002.

CUDDON, P.A. Electrophysiologic assessment of acute polyradiculoneuropathy in dogs: comparison with guillain-barré syndrome in people. J. Vet. Intern. Med., v.12, p.294-303, 1998.

CUMMINGS, J.F.; LAHUNTA, A.; HOLMES, D.F.; SCHULTZ, R.D. Coonhound paralysis: further clinical studies and eléctron microscopic observations. Acta Neuropathol., v.56, p.163178, 1982.

FEITOSA, M.M.; LARSSON, M.H.M.A.; USHIKOSHI, W.S.; PERRI, S.H.V. Determinação da velocidade de condução nervosa motora dos nervos radial e ulnar de cães clinicamente sadios. Arq. Bras. Med. Vet. Zootec., v.52, p.185-190, 2000.
KURIAN, M.A.; KING, M.D. Antibody positive myasthenia gravis following treatment with carbamazepine. neuropediatrics, v.34, p.276-277, 2003.

LIFSHITZ, M.; GAVRILOV, V.; SOFER, S. Signs and symptoms of carbamazepine overdose in young children. Pediatr. Emerg. Care, v.16, p.26-27, 2000 .

SAMPAIO, M.J.; FIGUEIROA, S.; TEMUDO, T. et al. Síndrome de Guillain-Barré em idade pediátrica. Protocolo de actuação. Acta Pediatr. Port., v.42, 33-42, 2011.

SITGES, M.; CHIU, L.M.; REED, R.C. Effects of levetiracetam, carbamazepine, phenytoin, valproate, lamotrigine, oxcarbazepine, topiramate, vinpocetine and sertraline on presynaptic hippocampal $\mathrm{Na}+$ and $\mathrm{Ca} 2+$ channels permeability. Neurochem. Res., v.41, p.758-769, 2016.

STANCIU, G.D.; MUSTEAȚĂ, M.; ARMAȘU, M.; SAFTENCU, P.M.; SOLCAN, G. Electrophysiological aspects in idiopathic acute canine Polyradiculoneuritis. Bull. Univ. Agricul. Sci. Vet. Med. Anim., v.71, p.2, 2014.

VOLK, H.A. International Veterinary Epilepsy Task Force consensus reports on epilepsy definition, classification and terminology, affected dog breeds, diagnosis, treatment, outcome measures of therapeutic trials, neuroimaging and neuropathology in companion animals. BMC Vet. Res., v.11, p.1-2, 2015. 